\title{
Disks in Young Binary Systems: Unresolved Millimeter-Wave Observations
}

\author{
Eric L. N. Jensen \\ Swarthmore College, Dept. of Physics \& Astronomy, \\ Swarthmore, PA 19081 USA
}

\begin{abstract}
Observations at millimeter and submillimeter wavelengths are sensitive to the total mass of circumstellar and circumbinary dust in a multiple system, and in some cases single-dish observations can help constrain the location of disk material in spite of their lack of spatial resolution. Young binary stars show a great diversity of disk properties, with a large part of the variation accounted for by binary separation. Many of the closest binaries (those with separations $a$ less than a few AU) harbor massive circumbinary disks. Binaries with $a \gtrsim 100 \mathrm{AU}$ tend to have massive circumstellar disks. In both cases, the properties of these disks (as deduced from millimeter and infrared fluxes) are indistinguishable from those around single stars. In the intermediate separation range (10 $\lesssim a \lesssim 100$ ), however, while disks do exist in most binaries, they are strongly limited by the presence of stellar companion, with inferred dust masses of order an Earth mass. While comparison of sample properties is secure, calculating masses in individual systems is limited by the uncertainty in dust opacity and surface density distribution laws (as in single stars), with the additional complication of the uncertain disk geometry in the system.
\end{abstract}

\section{Introduction}

The decade of the 1990 s opened with a widespread conviction among astronomers, based on abundant evidence from unresolved millimeter and infrared observations, that disks around young stars must exist. Ten years later, the advent of high-spatial-resolution imaging capabilities at optical, infrared, and millimeter wavelengths has led us to a point where there is overwhelming evidence from direct images that disks do exist (see reviews by Wilner \& Lay 2000; Ménard \& Stapelfeldt, this volume; and Dutrey \& Guilloteau, this volume). While the next decade will clearly belong to such resolved disk observations, particularly after the construction of the Atacama Large Millimeter Array (ALMA), at present the abundance and consistency of unresolved (i.e. single-dish) millimeter and infrared wavelength observations lend themselves best to characterizing some of the properties of disks around young stars in a statistical sense. In this review I will discuss what has been learned from these unresolved observations about the properties (especially masses) of disks in young binary systems. In particular, I will focus on observed differences between disks in binary systems and those around single stars. 


\section{Millimeter and Submillimeter Emission}

Observations at millimeter and submillimeter wavelengths are well suited for detecting disks, since the disk emission exceeds that of the stellar photosphere(s) by several order of magnitude. This is also true at mid-infrared wavelengths, but the higher opacity in the infrared means that the disk emission there is more likely to be optically thick. In the millimeter, at least the outer parts of the disk are believed to be optically thin for all but the most massive disks. Thus, the disk mass can be estimated by comparing the observed disk emission to that produced by a disk model. I discuss the problem of estimating disk masses in more detail in Section 4.

\subsection{Molecular Line Observations}

The millimeter and submillimeter part of the electromagnetic spectrum is rich with molecular transitions. Molecular line observations of disks can yield valuable information about disk composition, gas density, and depletion of molecular species from the gas phase onto dust grains.

To date, the most sensitive observations of molecular emission from disks in young binaries are those of Dutrey et al. (1997), who detected a number of different molecular species from the young binary GG Tau (Figure 1). They also observed the single star DM Tau and found results substantially similar to those for GG Tau. A broad implication of this work is that there are no obvious differences between the observed molecular properties of the disks around the binary and the single star. At present there have not been sensitive observations of a sufficiently large sample of stars to allow detailed comparison of the gas properties in disks around single stars to those around binaries.

\subsection{Continuum Observations}

Though molecular gas is thought to make up 99\% of the mass in disks around young stars (e.g, Liseau et al. 1995), it is the dust that contributes most of the continuum opacity. Thus, millimeter and submillimeter continuum observations provide a tracer of the dust around young stars. The differences in continuum emission between close binaries, wide binaries, and single stars have provided us with rich information about disks in the binary environment and will occupy the remainder of this review.

\section{Millimeter Flux as a Function of Binary Separation}

\subsection{The Observations}

Though theoretical investigations had long suggested that binary stars might truncate their disks (e.g., Lin \& Papaloizou 1979), it was the advent of sensitive surveys at millimeter wavelengths that provided the first observational evidence of such truncation. Beckwith et al. (1990) observed 86 young stars in the Taurus-Auriga star forming region at a wavelength of $\lambda=1.3 \mathrm{~mm}$. They noted that $75 \%$ of the 12 known binaries in their sample with separations less than $140 \mathrm{AU}$ were undetected at $1.3 \mathrm{~mm}$, compared to a $42 \%$ detection rate in the sample as a whole. With the great increase of the rate of discovery of young 


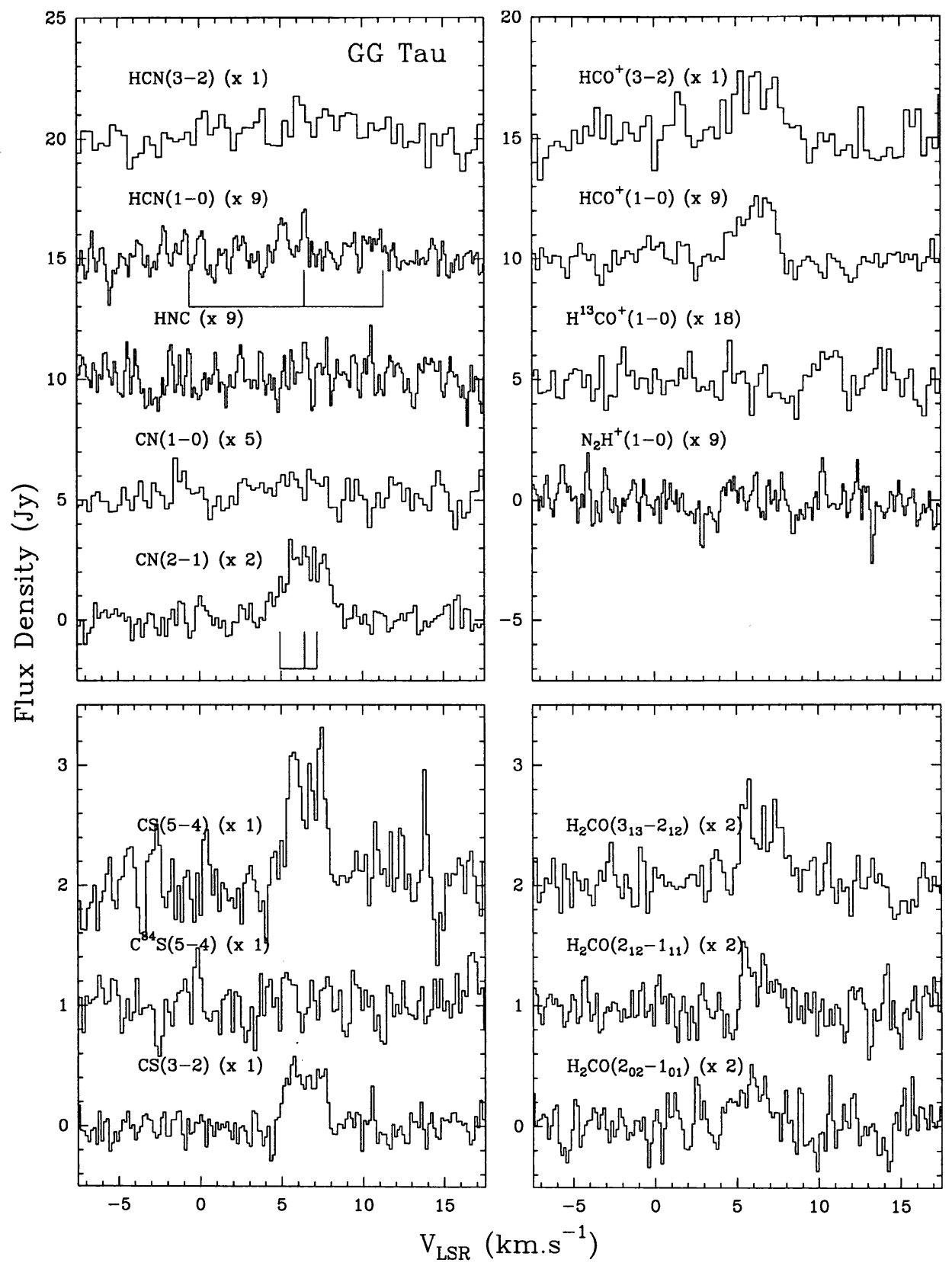

Figure 1. Molecular line spectrum of the young binary GG Tau, showing the abundance of molecules detected in the circumstellar and/or circumbinary environment (Dutrey et al. 1997). 
binaries in the 1990s, it was soon realized that there were far more than 12 close binaries in the Beckwith et al. sample. Coupled with additional millimeter and submillimeter observations, the expanded binary sample allowed analysis of the flux distributions of single and binary stars in samples large enough to yield statistically significant conclusions. Detailed studies were conducted by Koresko et al. (1993), Jensen, Mathieu, \& Fuller (1994, 1996b), and Osterloh \& Beckwith (1995); the effect was also noted by Simon et al. (1995), Nürnberger et al. (1998, and Henning et al. (1994).

Figure 2 shows the millimeter fluxes for binaries in Taurus-Auriga and Scorpius-Ophiuchus as a function of projected binary separation. There is a clear tendency for binaries with separations less than about $100 \mathrm{AU}$ to have low millimeter fluxes. Some wider binaries also have low fluxes, but the distribution of fluxes among the wide binaries is clearly different, with a mean at a higher flux, than that of the close binaries. This is more clearly seen in Figure 3, which shows the flux distributions for the two samples of binaries as well as that of the single stars. Because some of the systems are undetected at millimeter wavelengths, the distributions have been estimated using the KaplanMeier estimator, which takes upper limits into account in determining the most likely underlying distribution for a sample containing both detections and limits (Feigelson \& Nelson 1985). The close binaries have a different distribution of fluxes than either the wide binaries or the single stars with a confidence level of $>99.9 \%$, while the wide binaries and single stars are consistent with being drawn from the same distribution.

The observational result is quite robust. As noted above, a number of different authors have seen the same effect in the Taurus-Auriga data using a variety of analysis techniques. Jensen et al. (1996b) found the effect with high confidence levels in two independent samples of young binaries (Taurus-Auriga and Scorpius-Ophiuchus). In a smaller sample, Henning et al. (1994) found some evidence of the effect among Herbig Ae/Be stars.

\subsection{Disk Truncation}

These results are straightforwardly interpreted in the context of disk truncation in binary systems. Stable orbits for disk material exist only relatively near the stars (in circumstellar disks) or relatively far from either star (in a circumbinary disk outside the binary orbit). The region between these two disks is expected to be relatively unpopulated by dust or gas (e.g., Lin \& Papaloizou 1993; but see Artymowicz \& Lubow 1996). The exact range of stable orbits depends on the binary mass ratio and orbital eccentricity, but the cleared regions of the disk are approximately $0.5 a-2 a$ for circular orbits, or $0.2 a-3 a$ for eccentric orbits (where $a$ is the binary separation; Artymowicz \& Lubow 1994). If this is the case, then binaries with separations of tens of AU will clear a large fraction of their disks. As a representative example, the median binary separation among nearby G stars in the sample of Duquennoy \& Mayor (1991) is about 40 AU, and the mean eccentricity is 0.3 . Such a binary would have circumstellar disks of radius $8 \mathrm{AU}$ around each star, and a circumbinary disk beginning at $120 \mathrm{AU}$. If a typical disk radius is of order $100 \mathrm{AU}$, the system might have no circumbinary material at all. In contrast, a binary wider than a few hundred AU could harbor two undisturbed, 100-AU-radius circumstellar disks. Comparing these two disks 


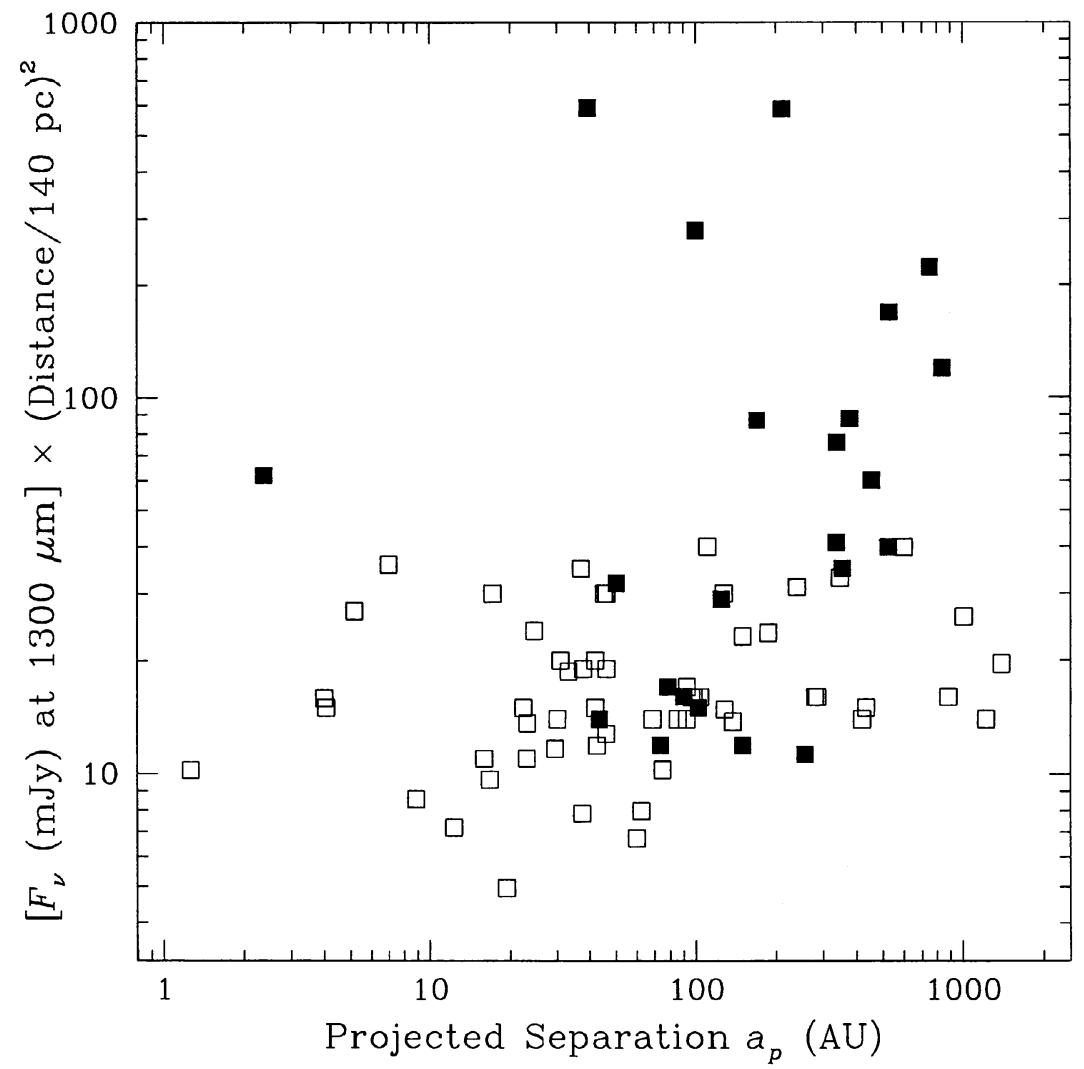

Figure 2. Millimeter flux as a function of projected binary separation for young binaries in Taurus-Auriga and Scorpius-Ophiuchus (adapted from Jensen et al. 1996b; no triple systems are shown). Filled symbols represent detections, while open symbols represent $3 \sigma$ upper limits. Binaries with separations less than $100 \mathrm{AU}$ tend to have low millimeter fluxes.

to the two 8-AU disks in the closer binary makes it clear why the millimeter emission from the closer binaries is so low.

To test this picture, Jensen et al. (1996b) calculated a simple model in which the disks in all binary systems are assumed to be the same, with the only difference being clearing of different amounts of the disks by binaries with different separations. Though this model is too simple to account for the range of fluxes seen at any given separation, it does broadly mimic the drop in fluxes seen at separations less than 100 AU in Figure 2 (see also Jensen et al. 1996b, Figure 4). Interestingly, this simple model also predicts that binaries with very small separations ( $a \simeq$ a few AU or less) should have substantial circumbinary disks. The sample discussed here does not include systems with separations less than $1 \mathrm{AU}$; we return to discussion of these systems and of circumbinary disks in Section 3.4. 
Kaplan-Meier estimator of $\lambda=1.3 \mathrm{~mm}$ flux distributions

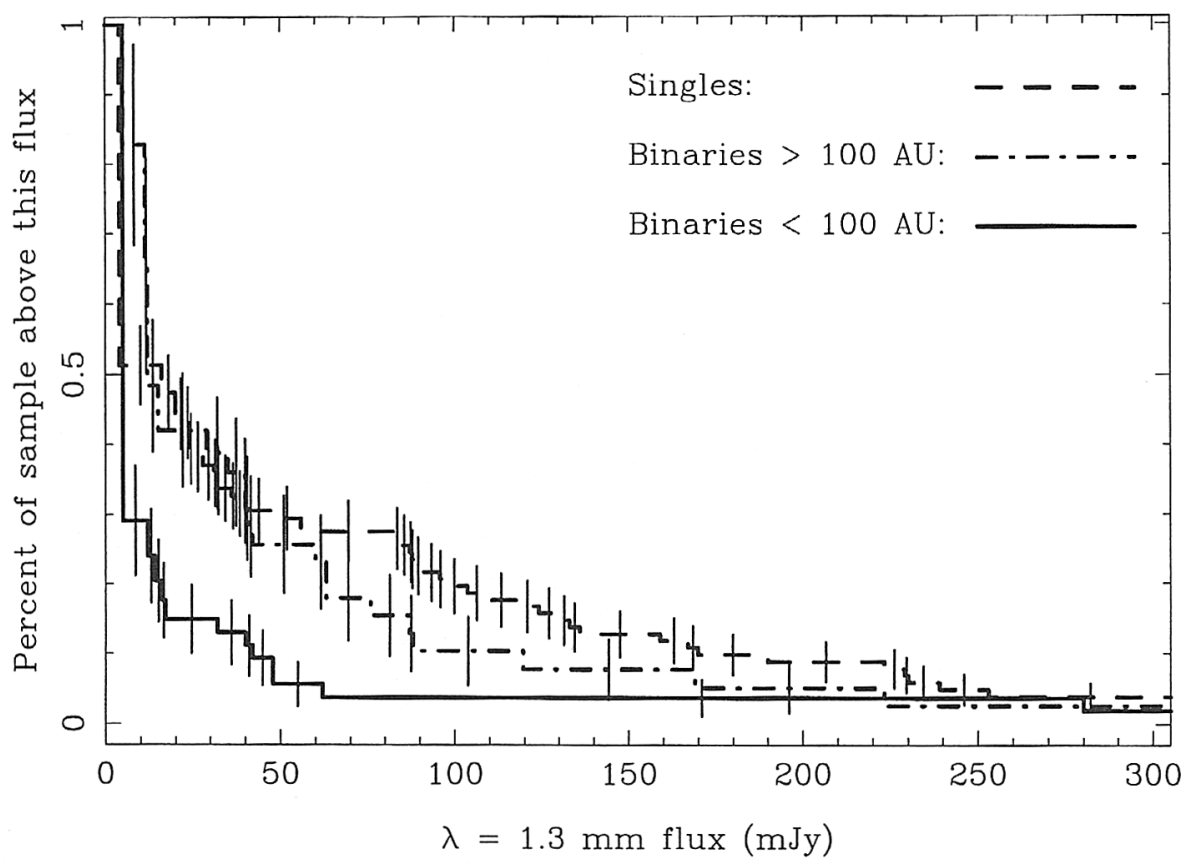

Figure 3. Kaplan-Meier estimator of the cumulative distributions of millimeter fluxes for single stars, binaries wider than $100 \mathrm{AU}$, and binaries closer than $100 \mathrm{AU}$. While the first two groups have indistinguishable flux distributions, the close binaries clearly have lower millimeter fluxes as a group.

This general picture of disks in binaries being unmodified at either very small or very large binary separations is confirmed by millimeter images of the quadruple system UZ Tau (Jensen, Koerner, \& Mathieu 1996a). A massive disk is seen around UZ Tau E, apparently undisturbed either by a spectroscopic binary in its center or by the presence of a companion (UZ Tau W) $500 \mathrm{AU}$ away. In marked contrast, UZ Tau W (itself a 50 AU binary) has a factor of four less millimeter emission than UZ Tau $\mathrm{E}$, consistent with the presence of only small circumstellar disks around its two stars.

\subsection{Infrared Fluxes and the Presence of Circumstellar Disks}

The preponderance of 10-100 AU binaries that are undetected at millimeter wavelengths (Figure 2) suggests, when considered by itself, that these systems might have no disks at all rather than simply having truncated disks. However, that is clearly not the case. Most of these systems were detected by IRAS at 12-100 $\mu \mathrm{m}$, indicating the presence of warm disk material close to one or both of the stars. Indeed, the distribution of $60 \mu \mathrm{m}$ fluxes of the close binaries is indistinguishable from that of the wider binaries or single stars (Jensen et al. 
1996b), indicating that the inner 5-10 AU (from which most of the infrared emission arises) of these disks is relatively undisturbed by the presence of a companion.

Or is it? What the preceding analysis tells us is that the inner disks in these systems most likely remain optically thick at $60 \mu \mathrm{m}$. Since the dust opacity at infrared wavelengths is much higher than at millimeter wavelengths, very little mass is needed to make the disk optically thick. Thus, while these inner disks could have surface densities that are comparable to those of disks around single stars or wider binaries, they could also have significantly lower surface densities. The observed infrared fluxes from binaries in the 10-100 AU separation range imply minimum disk masses of order $10^{-5} M_{\odot}$ (Jensen et al. 1996b). While these low disk masses are lower limits (since they assume partly optically thin emission), they may not be far from the truth. My collaborators and I have recently made sensitive submillimeter observations (using the SCUBA bolometer array on the James Clerk Maxwell Telescope) of some of the binaries in the 10100 AU separation range, yielding (in most cases) $3 \sigma$ upper limits of about 10 mJy on their fluxes at $\lambda=800 \mu \mathrm{m}$ (Mathieu et al., in preparation). These low fluxes imply upper limits of order $10^{-4} M_{\odot}$ on the disk masses. (Masses quoted here are subject to the caveats expressed in Section 4.)

\subsection{Circumbinary Disks}

As discussed above, a binary system may harbor up to three disks, two circumstellar and one circumbinary. Among binaries with separations of a few AU or less, there is little room for circumstellar disks, but circumbinary disks may be substantial. Though the number of known young binaries with such separations is small, the available evidence suggests that massive circumbinary disks may be common.

How can unresolved observations determine whether detected millimeter emission arises from within or outside the binary orbit? The argument is simple. Given a temperature $T$, an observed millimeter flux implies a minimum surface area $A$ for the emitting material since the emission from optically thick material simply scales with $A B_{\nu}(T)$. In a number of young, small-separation binaries, the entire area inside the binary orbit is too small to produce the observed millimeter flux, even if the orbit is filled with optically thick material at the photospheric temperature of the star! Thus, the bulk of the disk material must be in a circumbinary disk. This reasoning requires that circumbinary dișs be present in UZ Tau $\mathrm{E}$ (where the circumbinary disk is also resolved in millimeter interferometric images; Jensen et al. 1996a), DQ Tau, GW Ori, HP Tau, AK Sco, and V4046 Sgr.

Conversely, the lack of detections among the 10-100 AU binaries places sensitive limits on the amount of circumbinary material they might have. Even at the small-separation end of this range, circumbinary disks will lie at distances greater than 20-30 AU from the central stars, and thus the disks will be fairly cold. Assuming a minimum dust temperature of $15 \mathrm{~K}$ and optically thin emission, the typical millimeter flux upper limits place an upper limit of $\sim 0.005$ $M_{\odot}$ on the circumbinary disk masses in these systems (Jensen et al. 1996b). The notable exception is GG Tau, which has a massive, resolved circumbinary 
disk (see, e.g., Dutrey \& Guilloteau, this volume). Most 10-100 AU binaries, however, apparently have little circumbinary material.

\section{Disk Masses}

The disk masses quoted above were calculated using a standard model of a spatially flat disk, with assumed power-law surface density and temperature distributions as a function of radius (e.g., Beckwith et al. 1990). The only modification to this model to account for multiplicity is that the disks are cleared (i.e. surface density is zero) at the radii predicted by theory, 0.2 to 3 times the binary separation. This model is useful in order to get some estimate of disk masses, but it is important to understand its limitations.

The primary limitation (which applies to both binaries and single stars) is that the millimeter dust opacity is poorly known. Great progress has been made in directly measuring the millimeter opacity of dust in the laboratory, but the results vary widely depending on the grain composition, size distribution, and structure (Beckwith, Henning, \& Nakagawa 2000 and references therein). In working from observed fluxes (which depend on the optical depth $\tau_{\nu}$ ) to obtain disk properties, there is an unresolvable degeneracy between the normalization of the surface density power law $\Sigma_{0}$ (which is directly proportional to the disk mass) and value of the millimeter dust opacity $\kappa_{\nu}$, since $\tau_{\nu}(r)=\kappa_{\nu} \Sigma_{0}\left(r / r_{0}\right)^{-p}$. Thus, what we can truly constrain (assuming other parameters are well-known from other observations) is the product $\kappa_{\nu} \Sigma_{0}$ rather than the disk mass itself. Other parameters of the typical disk model (temperature distribution, exponent of the surface density distribution, inner and outer disk radii) may be uncertain as well, but improved spatial resolution and sensitivity of infrared and millimeter imaging are increasingly able to constrain these parameters, particularly when the observed images at different wavelengths are compared to model images (e.g., Lay, Carlstrom, \& Hills 1997; Koerner et al. 1998).

One assumption that may be reasonable for single stars but which seems problematic in the presence of a close companion is that of a smooth radial falloff in surface density (especially a single radial power law spanning a cleared gap). Again, I note that the increased spatial resolution of future millimeter observations will allow direct imaging of cleared gaps (see, e.g., Mundy, this volume) and much better constraints on the distribution of disk material in binary systems.

\section{Implications for Planet Formation in Binary Systems}

Since a majority of stars are members of binary systems, the properties of disks in the binary environment has important implications for the overall frequency of planet formation outside the solar system. Here I briefly examine the prospects of forming planets in binary systems in light of the discussion above. For broader discussions of this question, I refer the interested reader to other contributions in this volume, as well as Mathieu et al. (2000), from which some of the following arguments are adapted.

As noted above, most binaries retain at least some disk material. If these disks have surface densities comparable to those around single stars, and if 
planet formation is largely controlled by local conditions in the disk (i.e., a planet forming at $1 \mathrm{AU}$ from a star is insensitive to the disk conditions at 20 $\mathrm{AU})$, then the primary question becomes one of what fraction of binaries still have disks in the regions that are relevant for planet formation. In our own solar system, most of the planet formation has occurred in the range of 0.1-30 $\mathrm{AU}$ from the Sun. If we take the eccentric binary case cited above, where disks are cleared from $0.2 a$ to $3 a$, then any binary wider than about $150 \mathrm{AU}$ can have a 30-AU-radius circumstellar disks. Adopting the Duquennoy \& Mayor (1991) period distribution for main-sequence G stars, this includes roughly $33 \%$ of all binary systems. As noted above, many of the binaries with the lowest millimeter fluxes have circumstellar disks whose outer radii may be small but whose surface densities may be comparable to those around single stars. If we consider only the formation of terrestrial planets, disks with outer radii of only $\sim 5 \mathrm{AU}$ are required. This reduces the minimum binary separation to $25 \mathrm{AU}$ and thus includes $52 \%$ of all binaries. Finally, roughly $10 \%$ of binaries have $a<0.3 \mathrm{AU}$. As discussed in Section 3.4, many of these systems have massive circumbinary disks that begin at or inside $1 \mathrm{AU}$; such systems could form circumbinary planetary systems.

Finally, I note that we already know of planet formation in wide binary systems, since several of the known extrasolar planets orbit stars that are in binary systems (e.g., 16 Cyg B, 55 Cnc; Marcy, Cochran, \& Mayor 2000).

\section{Conclusions}

Are disks in binary systems like those around single stars? Yes and no. In that binary answer lie the two essential points of this review:

1. Disks are present in binary systems of all separations with the same frequency as in single stars.

2. Disk properties depend strongly on binary separation. The closest $(a<1$ $\mathrm{AU})$ and widest $(a>100 \mathrm{AU})$ binaries have disks, perhaps protoplanetary disks, that are similar to those around single stars. Intermediateseparation binaries, with separations comparable to a typical disk radius, clearly have less disk material, perhaps not even enough to form terrestrial planets.

Acknowledgments. I gratefully acknowledge the support of the National Science Foundation's Life in Extreme Environments program through grant AST 97-14246. I thank Allyn Dullighan for assistance with calculating disk masses.

\section{References}

Artymowicz, P., \& Lubow, S. H. 1994, ApJ, 421, 651

Artymowicz, P., \& Lubow, S. H. 1996, ApJ, 467, L77

Beckwith, S. V. W., Henning, T., \& Nakagawa, Y. 2000, in Protostars and

Planets IV, ed. V. Mannings, A. P. Boss \& S. S. Russell (Tucson:

University of Arizona Press), 533 
Beckwith, S. V. W., Sargent, A. I., Chini, R. S., \& Güsten, R. 1990, AJ, 99, 924 Duquennoy, A., \& Mayor, M. 1991, A\&A, 248, 485

Dutrey, A., \& Guilloteau, S. 2001, this volume

Dutrey, A., Guilloteau, S., \& Guélin, M. 1997, A\&A, 317, L17

Feigelson, E. D., \& Nelson, P. I. 1985, ApJ, 293, 192

Henning, T., Launhardt, R., Steinacker, J., \& Thamm, E. 1994, A\&A, 291, 546

Jensen, E. L. N., Mathieu, R. D., \& Fuller, G. A. 1994, ApJ, 429, L29

Jensen, E. L. N., Koerner, D. W., \& Mathieu, R. D. 1996a, AJ, 111, 2431

Jensen, E. L. N., Mathieu, R. D., \& Fuller, G. A. 1996b, ApJ, 458, 312

Koerner, D. W., Ressler, M. E., Werner, M. W., \& Backman, D. E. 1998, ApJ, $503, \mathrm{~L} 83$

Koresko, C. 1993, Ph.D. thesis, Cornell University

Lay, O. P., Carlstrom, J. E., \& Hills, R. E. 1997, ApJ, 489, 917

Lin, D. N. C., \& Papaloizou, J. 1979, MNRAS, 188, 191

Lin, D. N. C., \& Papaloizou, J. 1993, in Protostars and Planets III, ed. E. H. Levy \& J. I. Lunine (Tucson: University of Arizona Press), 749

Liseau, R., Lorenzetti, D., Molinari, S., Nisini, B., Saraceno, P., \& Spinoglio, L. 1995, A\&A, 300, 493

Marcy, G. W., Cochran, W. D. \& Mayor, M. 2000, in Protostars and Planets IV, ed. V. Mannings, A. P. Boss \& S. S. Russell (Tucson: University of Arizona Press), 1285

Ménard, F., \& Stapelfeldt, K. 2001, this volume

Nürnberger, D., Brandner, W., Yorke, H. W., \& Zinnecker, H. 1998, A\&A, 330, 549

Osterloh, M., \& Beckwith, S. V. W. 1995, ApJ, 439, 288

Simon, M., Ghez, A. M., Leinert, Ch., Cassar, L., Chen, W. P., Howell, R. R., Jameson, R. F., Matthews, K., Neugebauer, G., \& Richichi, A. 1995, ApJ, 443, 625

Wilner, D. J., \& Lay, O. P. 2000, in Protostars and Planets IV, ed. V. Mannings, A. P. Boss \& S. S. Russell (Tucson: University of Arizona Press), 509 